\title{
BMJ Open Effectiveness of workplace active rest programme on low back pain in office workers: a stepped-wedge cluster randomised controlled trial
}

\author{
Yamato Tsuboi (D) , ${ }^{1,2}$ Tomohiro Oka, ${ }^{1,3}$ Kiyomasa Nakatsuka, ${ }^{4}$ Tsunenori Isa, ${ }^{1}$ \\ Rei Ono (1) ${ }^{4}$
}

To cite: Tsuboi Y, Oka T, Nakatsuka K, et al. Effectiveness of workplace active rest programme on low back pain in office workers: a steppedwedge cluster randomised controlled trial. BMJ Open 2021;11:e040101. doi:10.1136/ bmjopen-2020-040101

- Prepublication history and additional supplemental material for this paper are available online. To view these files, please visit the journal online (http://dx.doi.org/10.1136/ bmjopen-2020-040101).

Received 29 May 2020 Accepted 17 May 2021

Check for updates

(C) Author(s) (or their employer(s)) 2021. Re-use permitted under CC BY-NC. No commercial re-use. See rights and permissions. Published by BMJ.

${ }^{1}$ Department of Community Health Sciences, Kobe University Graduate School of Health Sciences, Kobe, Japan

${ }^{2}$ BackTech, Kyoto, Japan ${ }^{3}$ Department of Rehabilitation, Anshin Hospital, Kobe, Japan ${ }^{4}$ Department of Public Health, Kobe University Graduate School of Health Sciences, Kobe, Japan

Correspondence to

Dr Rei Ono;

ono@phoenix.kobe-u.ac.jp

\section{ABSTRACT}

Objective This study aimed to investigate the effectiveness of workplace active rest programme (WARP) on chronic low back pain (LBP) among office workers. Design A closed cohort, stepped-wedge cluster randomised trial was conducted. The total duration of the study was 16 weeks (4 weeks for each step). Sequence allocation was randomised, but no one was blinded.

Setting This study was conducted in three offices in a Japanese electronics company. One office was for the administrative department, the others are for the engineering department.

Participants We recruited 29 office workers with LBP greater than 3 months. LBP due to specific injury or disease was excluded. The median age was 38 years, and $26(90 \%)$ were male. All participants completed the study. Interventions In the intervention phase, participants performed WARP comprising frequent stand-up and individualised brief exercise/physical activity during work. Physical therapists held an LBP workshop and developed tailor-made programmes before introducing WARP. We instructed participants to perform WARP at five timings during work. Control phase was set before the intervention and participants stayed as usual.

Primary and secondary outcome measures The primary outcome was pain intensity of LBP assessed using the Brief Pain Inventory. The secondary outcomes were work productivity loss measured using the Work Limitations Questionnaire, LBP disability assessed using the Roland-Morris Disability Questionnaire, psychosocial subscale assessed using the STarT Back Screening Tool and physical activity measured using triaxial accelerometers. These outcomes were collected at baseline and at 4-month follow-up evaluation.

Results In the intention-to-treat analysis, WARP did not show any significant effects on pain intensity $(\beta, 0.01$; $95 \% \mathrm{Cl}-0.50$ to 0.52 ) and on the secondary outcomes. The median adherence to WARP was $28.6 \%$ (IQR, 16.841.1), which was equal to 1.43 times per day. No adverse effect was observed.

Conclusions The present study was unable to confirm the effectiveness of active rest in improving LBP. Hence, further study needs to investigate its effectiveness.

Trial registration number UMIN000033210.
Strengths and limitations of this study

- This study is the first pragmatic trial conducted in a real-world setting that investigates the feasibility and effectiveness of active rest.

- All participants completed the workplace active rest programme (WARP).

- Adherence to WARP was lower than expected.

- Because recruited office workers had relatively mild low back pain, we were unable to confirm whether WARP is effective among office workers with severe low back pain.

\section{INTRODUCTION}

Low back pain (LBP) is a prevalent health problem among office workers ${ }^{12}$ and is the leading cause of decreasing healthy life expectancy worldwide. ${ }^{3}$ Moreover, LBP results in a large socioeconomic burden due to work productivity loss and medical expenses. ${ }^{45}$ In terms of both individual and social impact, LBP among office workers is a crucial problem which should be tackled.

Office workers are workers who stay in prolonged sitting position during most of their working time. ${ }^{6}$ Prolonged sitting is one of the causes of LBP, which is also due to several factors such as increased disc pressure, $^{8}$ decreased trunk mobility ${ }^{9}$ and less posture variation. ${ }^{10}$ Although previous studies have investigated the effectiveness of ergonomic intervention and back support, these are considered ineffective in improving LBP. ${ }^{11}{ }^{12}$ Recently, the use of standing desk has been shown to be effective in improving $\mathrm{LBP},{ }^{13}$ but it has the following limitations: it requires a lot of space and is costly. Therefore, easy-to-use solutions are required in the workplace.

Active rest (taking a break with exercise/ physical activity in the workplace) could possibly improve LBP because it has the 
following characteristics: (1) sedentary break by standing up, which can prevent prolonged sitting; and (2) exercise/physical activity, which is recommended in the LBP guidelines. ${ }^{1415}$ A previous study showed that officebased stretching (10-15 min/session, 3 times/week) was effective in reducing the occurrence of musculoskeletal discomfort when compared with no intervention. ${ }^{16}$ However, in our study, we developed a shorter exercise programme involving frequent sessions (a few minutes per session, five times per day, except on weekends) because we aimed to promote frequent standing to break the habit of prolonged sitting. Although a positive effect of active rest on LBP has been shown in a laboratory study, ${ }^{17}$ its effectiveness in the real-world setting is still unknown. We hypothesised that there is a difference in effectiveness between laboratory and real-world setting. Thus, the present study aimed to investigate the effectiveness of the workplace active rest programme (WARP) on chronic LBP and work productivity loss among office workers in a real-world setting.

\section{METHODS}

\section{Study design}

The present study was conducted according to the extension of the Consolidated Standards of Reporting Trials 2010 statement for stepped-wedge cluster randomised controlled trial (SW-CRT). ${ }^{18}$ We used a closed cohort SW-CRT involving randomisation of clusters to different sequences. SW-CRT is a crossover design with repeated measurement, in which clusters switch from control to intervention condition. SW-CRT is a suitable study design if we assume that the intervention will do more good than harm, hence making it unethical to withhold the intervention from a control group. Thus, because it is morally acceptable and beneficial for participant recruitment, we introduced the SW-CRT design. ${ }^{19}$ Moreover, this is the pragmatic design, which increases statistical power and decreases needed clusters compared with those in parallel CRT. ${ }^{20}$ The present clinical trial was registered with University Hospital Medical Information Network (UMIN) Clinical Trials Registry.

As figure 1 shows, we conducted the present study in three offices (clusters) in a Japanese electronics company. We set three sequences, where an office switched from the control condition to the intervention condition one by one. The total duration of the study was 16 weeks (4 weeks for each step). Evaluation was conducted at baseline and at four points during the last week of each step. Due to the study's closed cohort design, participants assessed in different periods were the same participants.

\section{Patient and public involvement}

Office workers with LBP were not involved in developing the research question, but we consulted them about the design of the study (especially the intervention programme) in terms of feasibility and applicability by joining the employees' health committee. During the trial, they helped us to hold an LBP workshop by arranging a room and equipment. We asked them to assess the burden of the intervention before they joined the study. We already disseminated the results of our study to the participants and reported them at the employees' health committee.

\section{Participants' recruitment}

We recruited 29 participants from three offices of a Japanese electronics company in July 2018. Three offices were separated from one another. First, participants were approached by the public health nurse working in this company. When they expressed interest in the study, the public health nurse introduced them to us. Subsequently, the researchers explained the study to the participants, and the participants provided informed consent for inclusion in the study.

Office workers were eligible for the present study if they have the following characteristics: (1) full-time workers (all workers worked in the same day shifts), (2) engaged in desk work greater than 4 hours/daily working time (selfreported ${ }^{21}$ and (3) had LBP for greater than 3 months.

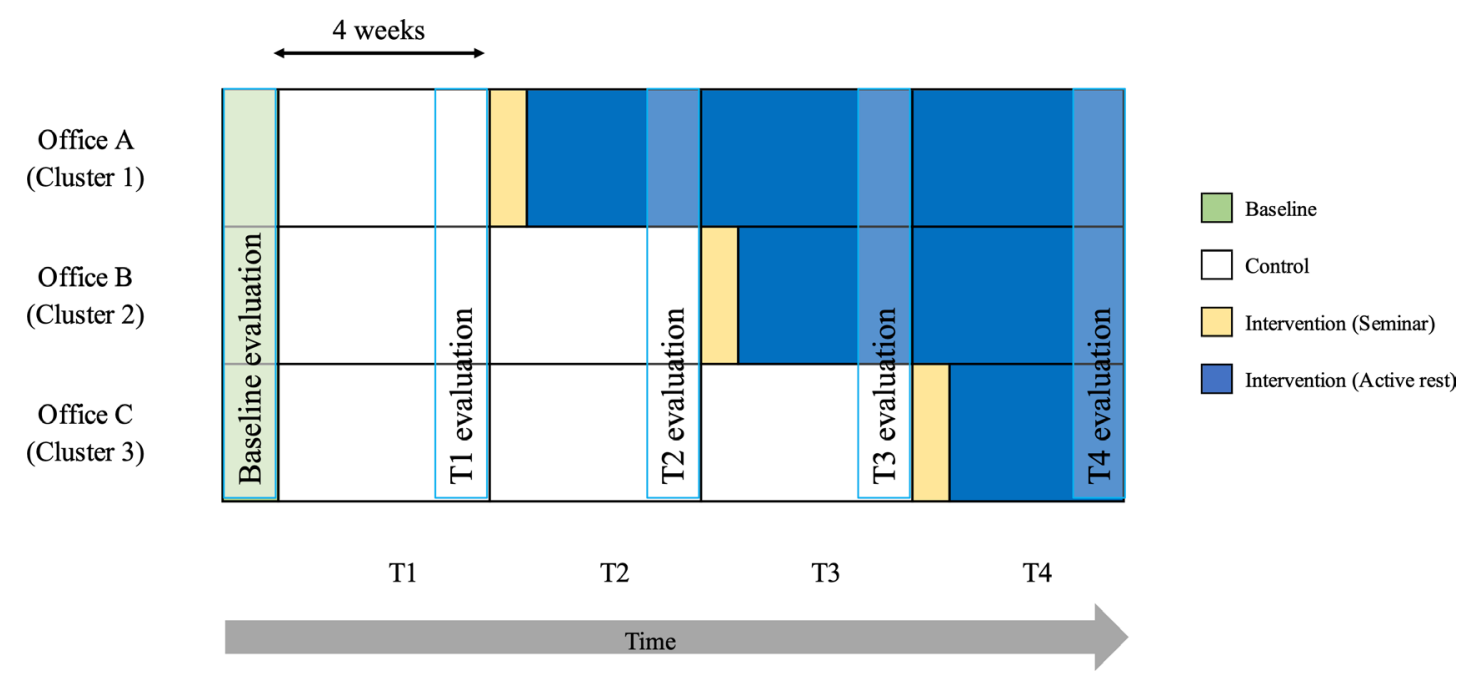

Figure 1 Diagram of stepped-wedge cluster randomised controlled trial design. 
The location of LBP was defined as pain between the 12th rib and the inferior gluteal folds. ${ }^{22}$ Exclusion criteria were as follows: (1) LBP caused by fracture and trauma injuries, infectious diseases and internal organ disorders and (2) difficulty participating in the study due to medical or surgical disease. Cluster-level eligibility criteria were as follows: (1) an office where most workers were engaged in desk work and (2) supervisors granting permission in the performance of the study. Whereas office A was for an administrative department, office $B$ and office $\mathrm{C}$ were for engineering department.

All participants provided written informed consent for inclusion in the study.

\section{Randomisation and blinding}

Offices were randomly assigned to one of the three successive sequences (one office per sequence) after all clusters and participants were recruited. A researcher who was not involved in the recruitment performed random allocation using computer-generated random numbers and coded information about offices. To prevent contamination, both clusters and participants were not informed of the time the intervention started and the detailed programme of the intervention until 2 weeks before the intervention started. We also asked the participants exposed to the intervention not to disclose the programme content to other workers. Due to the nature of the present study, participants, intervenient and outcome assessors (self-reported) could not be blinded. The data analyst was also not blinded to group allocation.

\section{Intervention}

In the intervention phase, we offered WARP in two parts. First, we held the LBP workshop (group), followed by the introduction of active rest in the workplace. The LBP workshop was held when the group moved from the control phase to the intervention phase.

The purposes of the LBP workshop were as follows: to allow the participants to understand LBP and sedentary behaviour, develop customised exercise programme and explain how WARP is performed after the workshop. The LBP workshop was held at the company's gymnastics room after work for $90 \mathrm{~min}$ by two or three physical therapists (PTs) (PTs with expertise in LBP, at least 3 or more experience years) including the primary researcher (YT). To avoid inconsistency on workshop contents in PTs, we discussed and agreed with its contents before workshop. We disseminated leaflets about the contents of the LBP workshop to the participants. First, we gave lecture on the following: (1) LBP causes and interventions using a biopsychosocial model and (2) the impact of sedentary behaviour on health (death, non-communicable diseases and LBP). Second, evaluation was performed using a physical examination and an interview sheet (a brief file is described in online supplemental figure 1). We evaluated trunk flexion and extension (comfortable direction), static trunk posture (sagittal plane, lordosis/ kyphosis), Thomas test (flexibility of the iliopsoas muscle), ${ }^{23}$ finger-floor distance test (spine and hip joint movement) and one-finger test (positive result indicates sacroiliac joint pain) ${ }^{24}$ and asked if the participants felt painful sensations when sitting or standing. Third, individualised exercise programmes were developed based on the results of the evaluation. Some exercises were recommended based on the results of the physical examination and interview sheets (online supplemental figures 1 and 2). We prepared six types of exercise focusing on spine and hip stretch and training, which can improve spine and hip joint mobility and decrease lumbar disc pressure (trunk extension exercise, stretching of the iliopsoas and hamstrings, abdominal oblique, erector spinae muscles, thoracolumbar fascia). We selected these exercises because these can be briefly performed by the participants when they stand up. We let them perform the recommended exercises during workshop after they had seen the demonstration. If participants had difficulty in performing the exercise, we individually helped them.

At the end of the workshop, we explained to the participants how and when WARP is performed. Participants were instructed to perform WARP at five timings (just before the work starts, morning break, lunch break, afternoon break, after the work is finished). A chime ringed at these five timings, and we asked them to stand up and perform their exercises for a few minutes after the chime ringed. We also recommended performing WARP other than the five fixed timings. However, the participants were not required to perform the programme. We explained the content of WARP and introduced some brief exercises to other workers in the same office. It enables participants to easily perform exercise at the workplace because they understand what they do. Additionally, to determine if problems occurred after performing WARP, researchers visited each office once a month.

\section{Control}

When the participants were in the control phase, we did not perform any intervention to the participants (usual work).

\section{Primary outcome}

The primary outcome was LBP intensity. We used the pain intensity subscale of the Brief Pain Inventory (BPI), which is well validated and reliable among patients with non-cancer pain including LBP. ${ }^{25}{ }^{26}$ BPI consists of four questions rating pain intensity separately at 'worst', 'least', 'average' and 'now' during the last 24 hours using 11-point Numerical Rating Scale (NRS), ranging from 0 (no pain) to 10 (worst imaginable pain). Finally, the mean of these four items was used as the BPI score: BPI score $=($ worst + least + average + now $) / 4$. A Japanese version of BPI has good validity and reliability. ${ }^{27}$

At the time of trial registration, although we had planned to evaluate weekly LBP intensity, we changed to once in 4 weeks evaluation. This is because weekly evaluation was not feasible at this company in terms of responders' burden in answering questionnaires. 


\section{Secondary outcome}

The Roland-Morris Disability Questionnaire (RDQ) is a validated 24-item questionnaire that assesses disability due to LBP, such as 'I change position frequently to try and get my back comfortable'. ${ }^{29}$ Each item is scored either 0 or 1 , with all scores summed to a total between 0 and 24 (a higher score indicates greater disability level).

The STarT Back Screening Tool is a validated screening tool that predicts future disability level. ${ }^{30} 31$ We used the five-item psychosocial subscale of the STarT Back Screening Tool, including fear of movement, depressive symptom, catastrophic attitude, anxiety and pain distress. Scores ranged from 0 to 5 (a higher score indicates higher possibility of future disability level).

The Work Limitations Questionnaire (WLQ) is a validated 25-item questionnaire that evaluates work productivity loss due to physical/psychological issues. ${ }^{32} 33$ The WLQ is composed of the following four subscales: (1) time management (difficulty in performing job tasks in a timely manner and in scheduling tasks); (2) mentalinterpersonal demands (difficulty in performing cognitive job tasks and in interacting with colleagues); (3) physical demands (ability to perform job tasks involving body strength, movement, endurance, coordination and flexibility); and (4) output demands (work quantity and quality reduction and timeliness of completed work). Additionally, 'not applicable' was also provided as a response option and treated as a missing value. All subscales scores were converted to percentage, from $0 \%$ (least limited) to $100 \%$ (most limited). Work productivity loss $(\%)$ was calculated from the weighed sum of the four subscale scores using a validated algorithm ranging from $0 \%$ to $24.9 \%$. A higher score indicates higher level of work productivity loss.

To measure physical activity and sedentary behaviour, we distributed triaxial accelerometers (Active style Pro HJA-750C, Omron Healthcare) to the participants during each step. Details of the accelerometer measurement procedure were described elsewhere. ${ }^{34} 35$ Participants were instructed to wear triaxial accelerometers on their waist during only working time for 5 days. Data were recorded in $60 \mathrm{~s}$ epoch. In addition to the number of steps, time spent in moderate-to-vigorous physical activity (MVPA, $\leq 3.0$ metabolic equivalent; METs), light physical activity $(1.5<$ MET <3.0) and sedentary behaviour (MET $\leq 1.5)$ was calculated using R V.3.5.2. Days with at least 4 hours of wearing time or $75 \%$ of working hours were considered a valid day, ${ }^{36}$ and we included the data with at least one valid day in the analysis. Non-wear time was defined as a period with continuous zero count lasting over $60 \mathrm{~min}$.

\section{Other measurements}

We collected demographic data such as age, sex, height, weight and body mass index. Participants were asked whether they were ever diagnosed with the following conditions: lumbar disc herniation, lumbar canal stenosis, lumbar compression fracture, trauma, spinal metastasis, fibromyalgia, rheumatoid arthritis and infectious spondylitis. Participants also reported the status of their analgesic administration (none, rarely, sometimes, often and always), consultation in orthopaedic clinics or complementary medicine for LBP (none, once, twice, three times, four times and greater than five), sleep quality (very good, fairly good, fairly bad and very bad), and other musculoskeletal pain including neck, shoulder, elbow, wrist, hip, knee and foot (NRS). At the final follow-up evaluation (T4 evaluation in figure 1), participants answered about their satisfaction (satisfied very much, satisfied, normal, dissatisfied, dissatisfied very much) and free opinion about WARP.

\section{Adherence}

To evaluate adherence to WARP, we asked participants to keep diaries on whether they performed WARP or not in each five timing. Adherence is calculated $100 \%$ if they performed WARP at all five timings during the whole intervention phase. Because WARP is a programme at the workplace, we did not include holidays when assessing adherence.

\section{Sample size}

We calculated the sample size using formula specific for stepped-wedge design. ${ }^{20}$ The primary outcome difference and SD were set as 2.0 and 2.5 , respectively. ${ }^{37}$ The following assumed parameters were used: cluster size $=10$, intracluster correlation coefficient $=0.05$, number of step $=3$, number of baseline measurement $=1$, measurement after each step $=1$, two-sided $\alpha$ level $=0.05$ and $\beta=80 \%$. To detect a 2-point difference in primary outcome, a total of 22 participants were needed. Considering dropout, we estimated 30 participants as the required sample size, and 29 participants actually joined the present study. Although we set cluster size as 10 before recruitment, the actual size of the two clusters was 8 . We conservatively performed sample size calculation by changing some parameters. However, the required sample size was not changed (22 participants) even if it is 8 participants. Therefore, this difference would not affect the results of our study.

\section{Statistical analysis}

For the characteristics of participants, categorical variables were presented as frequency and percentage and continuous variables as mean $\pm \mathrm{SD}$. If the distributions of the continuous variables were skewed, data were presented as median (range or IQR).

We performed both intention-to-treat (ITT) and perprotocol analyses to investigate both the effectiveness and the efficacy of WARP. The primary analysis was ITT analysis because this study aimed to investigate the pragmatic effectiveness of WARP in a real-world setting. Regarding ITT analysis, we performed linear mixed effect model for all outcomes, setting the intervention as the fixed effect, individual and office as the random effect, and calendar time as the confounding factor. Regarding per-protocol analysis, we also performed the linear mixed effect model 


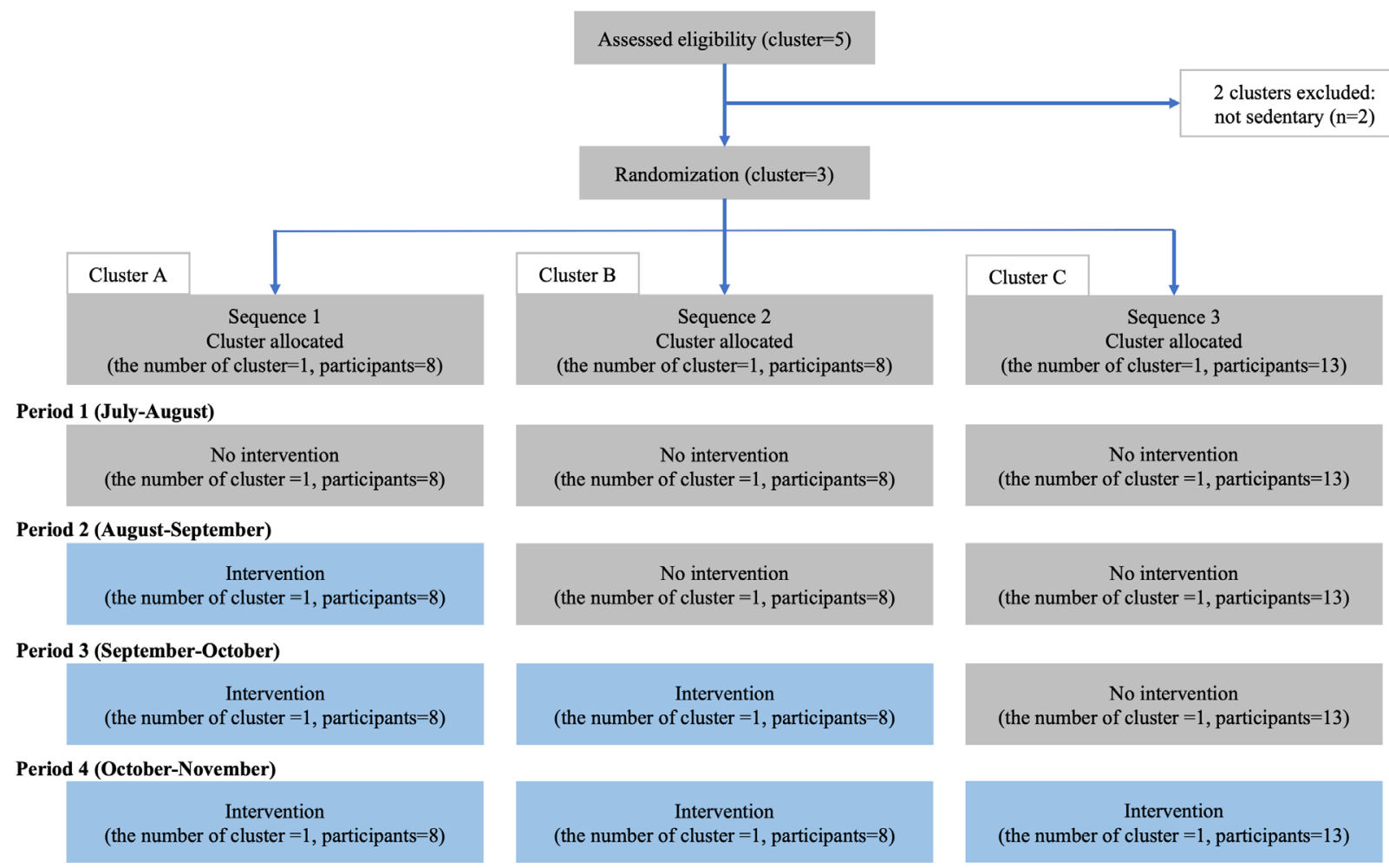

Figure 2 Flow chart for stepped-wedge cluster randomised trial.

for all outcomes after excluding participants whose adherence to WARP was $28.6 \%$ (median) or less. Unstandardised coefficients and $95 \%$ CIs were calculated.

All statistical analyses were performed using Stata/ IC V.15.1 software. $\mathrm{P}<0.05$ was considered statistically significant.

\section{RESULTS}

We recruited 29 office workers from three offices in July (figure 2). As planned, office A performed the intervention in the first period (August), office B in the second period (September) and office $\mathrm{C}$ in the third period (October). All participants continued WARP until the end (no dropout) of the study. Twenty-eight participants completed the baseline and each follow-up evaluation (T1-T4). Only one participant did not answer the T3 evaluation, but answered other evaluations.

The median age was 38 years, and $26(90 \%)$ were male (table 1 ). The median pain intensity assessed using BPI was 2.0 (IQR, 0.8-2.2), and the median score on RDQ was $1.0(0.0-2.0)$. Only two participants had clinic or alternative care, and only one participant often received analgesic medication. The median proportion of sedentary time was $79.6 \%$ (68.1-84.1). The median productivity loss estimated by WLQ was $2.2 \%(0.8-5.9)$. Regarding the difference in characteristics of the three offices, participants were younger in office $\mathrm{C}$ than in other offices. Pain intensity was lighter in office B than in other offices.

The median adherence to WARP was $28.6 \%$ (16.841.1), which is equal to 1.43 times per day (figure 3). Participants with higher adherence had relatively higher pain intensity, disability due to LBP and higher work productivity loss (online supplemental table 1) compared with those with lower adherence. Furthermore, low adherence was related to longer duration of WARP (adherence, office $\mathrm{A}<\mathrm{B}<\mathrm{C}$ ).

For ITT analysis with adjustment for time effects, pain intensity did not improve better in the intervention phase compared with the control phase $(\beta, 0.01 ; 95 \%$ CI -0.50 to 0.52 ) (table 2). Regarding secondary outcomes, no significant improvement was observed. For perprotocol analysis with adjustment for time effects $(n=14)$, time management demands and mental-interpersonal demands (WLQ subscale), MVPA improved better in the intervention phase compared with the control phase. $\mathrm{RDQ}$, productivity loss and step significantly improved better in the intervention phase compared with the control phase. Calendar time had significant or marginal significant positive effects on the primary and secondary outcomes. Any adverse effects were not reported in the present study.

Regarding participants' satisfaction with WARP, 4 (14\%) were very satisfied, $10(34 \%)$ were satisfied and 15 $(52 \%)$ were normal. No one was unsatisfied with WARP. As regards positive comments, some said the following: "I understood my back pain could be improved, and exercise was easy to perform," "It was nice to know effective stretch," "I feel my back pain is gradually improved," "I could be careful for prolonged sitting," "I want to make use of personalized exercise," "Back pain was gradually improved," "I could consider problems and methods for solving back pain," and "It was nice to undertake an 
Table 1 Characteristics of the participants

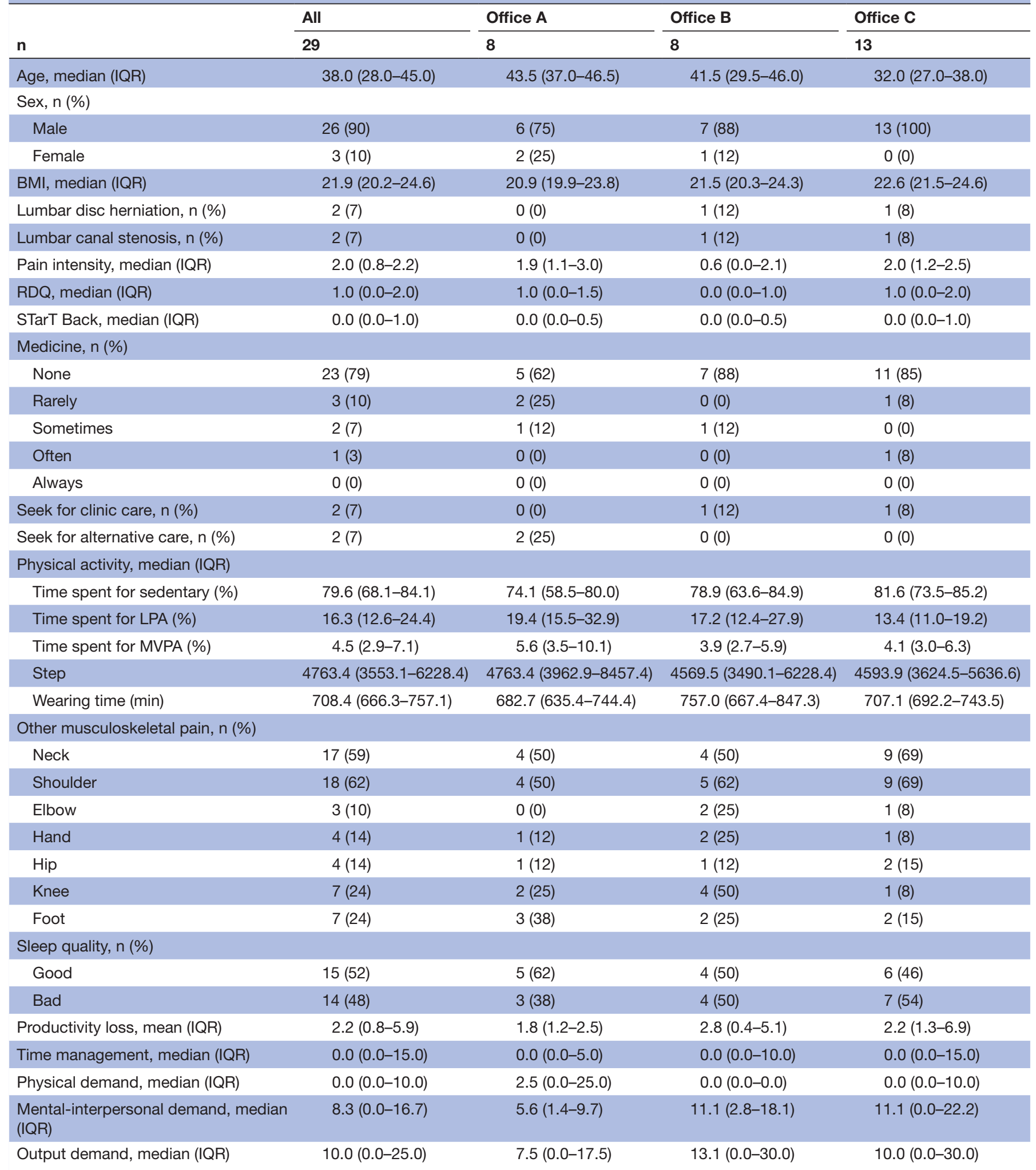

STarT Back, STarT Back Screening Tool; BMI, body mass index; LPA, Low physical activity; MVPA, Moderate-vigorous physical activity; RDQ, Roland-Morris Disability Questionnaire.;

exercise instruction from professionals." As regards negative comments, some said the following: "Not enough follow-up other than questionnaire," "Regular feedback based on follow-up data can motivate us to perform this program, but actually no feedback in this program," "There were few people doing exercise around me, so it was hard to do exercise," and "I wanted to know exercise during sitting." 
Median 28.6\% (IQR: $16.8-41.1)$

\begin{tabular}{ccccc} 
& & & Step3 & Step4 \\
\cline { 2 - 5 } & Step1 & Step2 & & $19.5 \%(11.6-22.1)$ \\
$\begin{array}{c}\text { Office A } \\
\text { (Cluster 1) }\end{array}$ & - & $23.6 \%(15.0-28.5)$ & $20.0 \%(12.9-44.3)$ & $25.7 \%(18.3-35.4)$ \\
$\begin{array}{c}\text { Office B } \\
(\text { Cluster 2) }\end{array}$ & - & - & $30.2 \%(24.1-46.0)$ & $33.3 \%(22.5-50.0)$ \\
\hline $\begin{array}{c}\text { Office C } \\
\text { (Cluster 3) }\end{array}$ & - & - & - & 3 \\
\hline
\end{tabular}

Data were shown in median (Interquartile range)

Figure 3 Adherence to the intervention among each step and office.

\section{DISCUSSION}

In summary, ITT analysis showed that WARP did not have significant positive effects on LBP intensity and other secondary outcomes such as LBP disability or work productivity. The median adherence to WARP was $28.6 \%$ (1.43 times per day), which was significantly lower than we expected. Per-protocol analysis revealed that WARP was not associated with LBP outcomes, but WARP had significant positive effects on some subscales of work productivity (time management demands, mental-interpersonal demands) and MVPA.

Although a recent systematic review investigated the current evidence on active rest, they concluded that there was low-quality evidence for the conflicting effectiveness in $\mathrm{LBP}^{38}$ Studies included in the systematic review were conducted in the laboratory setting or healthy subjects without LBP. Therefore, this is the first randomised controlled trial that investigates the effectiveness of active rest in LBP and work productivity in a real-world setting. However, we were not able to demonstrate the significant positive effect of WARP on LBP. While the present study evaluated the effect of short and frequent office-based exercises (a few minutes per session, five times per day, except weekends) on LBP symptom reduction, a previous study showed the effect of long and less frequent officebased exercises (10-15 min per session, 3 times per week) on LBP symptom reduction. ${ }^{16}$ These differences between the two study designs should be considered when interpreting the results of our study.

We have two potential explanations about the negative results of our studies. First, it might be due to low adherence to WARP, which could diminish its efficacy. Although we considered some strategies to keep adherence (eg, introducing WARP to all workers other than

Table 2 Intervention effect on each outcome

\begin{tabular}{|c|c|c|c|c|c|c|}
\hline & \multicolumn{3}{|c|}{ ITT analysis $(n=29)$} & \multicolumn{3}{|c|}{ Per-protocol analysis $(n=14)$} \\
\hline & $\beta$ & $95 \% \mathrm{Cl}$ & $P$ value & $\boldsymbol{\beta}$ & $95 \% \mathrm{Cl}$ & $P$ value \\
\hline Pain intensity & 0.01 & -0.50 to 0.52 & 0.965 & -0.16 & -0.90 to 0.58 & 0.680 \\
\hline \multicolumn{7}{|l|}{ WLQ } \\
\hline Productivity loss (\%) & -1.04 & -2.70 to 0.61 & 0.218 & -2.31 & -4.79 to 0.17 & 0.068 \\
\hline Mental-interpersonal demands & -5.31 & -11.10 to 0.48 & 0.072 & -10.48 & -20.56 to -0.41 & 0.041 \\
\hline Physical demands & 1.23 & -2.78 to 5.25 & 0.548 & 1.92 & -3.86 to 7.71 & 0.515 \\
\hline Output demands & -1.05 & -8.61 to 6.52 & 0.786 & -9.34 & -21.88 to 3.19 & 0.144 \\
\hline \multicolumn{7}{|l|}{ Physical activity } \\
\hline Time spent sedentary (\%) & -0.95 & -4.58 to 2.67 & 0.607 & -1.80 & -6.62 to 3.03 & 0.466 \\
\hline Step & 146.80 & -850.72 to 1144.33 & 0.773 & 889.44 & -511.34 to 2290.21 & 0.213 \\
\hline STarT Back total score & -0.20 & -0.57 to 0.18 & 0.306 & -0.41 & -1.08 to 0.27 & 0.235 \\
\hline
\end{tabular}

All models were adjusted with time effect; participants with less than $28.6 \%$ (median) or the median for adherence were excluded from the per-protocol analysis. All outcomes were measured at five time points (once in 4 weeks).

ITT, intention-to-treat; LPA, low physical activity; MVPA, moderate-vigorous physical activity; RDQ, Roland-Morris Disability Questionnaire; WLQ, Work Limitations Questionnaire. 
the participants of this study in the same office, ringing the chime to inform them of WARP timing, and tailormade exercise programme), these might be insufficient to improve adherence. Previous studies suggested supervised exercise and group-based exercise. ${ }^{39}$ However, there were no strict supervision or group-based exercises in our study because we tried to investigate the effectiveness of a pragmatic easy-to-use solution. Moreover, lower adherence to workplace exercise was influenced by poorer psychosocial work environment (eg, influence at work, work pace, quantitative demands, interpersonal relations) and lower exercise self-efficacy. ${ }^{40} \mathrm{~A}$ further study should be conducted to perform such strategies to improve adherence, but simplicity and acceptance from the employee and the employer should be considered in terms of practical use. The second potential explanation for the negative results is that the participants in our study had lower level of LBP intensity at baseline, which leads to low motivation for WARP and floor effect. Actually, participants with lower LBP intensity had lower adherence than those with high LBP intensity. We considered the floor effect owing to the mild pain by specifically recruiting workers with back pain (NRS was 3 or higher). However, a time lag between recruitment and baseline assessments due to coordinating the schedule of the LBP workshop might have led to a decrease in pain levels at the time the study was actually conducted. Future studies should focus on the fluctuations of outcome variables between recruitment and baseline assessments.

Regarding per-protocol analysis, unstandardised coefficients of most outcome parameters were significantly positive compared with those of the ITT analysis. A previous study reported that active rest $(10 \mathrm{~min}$ fitness programme at lunch break) has positive effects on vigour, interpersonal stress and physical activity. ${ }^{41}$ Although the results of the per-protocol analysis should be carefully interpreted owing to selection bias and an underpowered analysis, these results indicate that WARP could have positive effects if its adherence was ideally kept.

Several limitations should be considered when interpreting the results of our study. First, adherence to the programme was very low, which might lead to underestimation of the potential efficacy of WARP. Second, severity level of LBP was relatively mild in this population, which might cause floor effect especially for BPI and RDQ. We should have set the inclusion criteria about the severity level of LBP to eliminate the floor effect. Otherwise, if mild LBP is common in the working population compared with primary care, we should focus on the incidence or recurrent incidence of LBP in terms of primary prevention. Finally, owing to the limited number of workplace settings and types included within one company, the results of the study should not be considered generalisable to other workplace settings.

We were unable to conclude that active rest is effective in LBP and productivity loss from the results of the present study. However, the present study provided valuable information for conducting similar research, although the strategies implemented in this study might be insufficient to maintain adherence. In the future, we need to study its effectiveness with high adherence or among workers with higher level of LBP intensity.

Acknowledgements We would like to thank all participants who willingly joined in our study. We also gratefully acknowledge the public health nurse, Ms Sato, who helped us in the recruitment and data collection. We appreciate the advice of Professor Omori from the Clinical and Translational Research Center of Kobe University Hospital regarding our research protocol.

Contributors $\mathrm{YT}, \mathrm{TO}, \mathrm{KN}, \mathrm{Tl}$ and RO contributed to the conception and design of the study. YT, KN and TI conducted recruitment, intervention, data collection and data analysis. YT wrote the first draft of the article, and TO, KN, TI and RO revised it and agreed to the final paper.

Funding This work was supported by Meiji Yasuda Life Foundation of Health and Welfare.

Competing interests None declared.

Patient consent for publication Not required.

Ethics approval The study was approved by the Ethics Committee of Kobe University Graduate School of Health Sciences (no. 718).

Provenance and peer review Not commissioned; externally peer reviewed.

Data availability statement Data are available upon reasonable request. Data, STATA code for statistical analyses and R code for data processing of accelerometers are available upon reasonable request.

Supplemental material This content has been supplied by the author(s). It has not been vetted by BMJ Publishing Group Limited (BMJ) and may not have been peer-reviewed. Any opinions or recommendations discussed are solely those of the author(s) and are not endorsed by BMJ. BMJ disclaims all liability and responsibility arising from any reliance placed on the content. Where the content includes any translated material, BMJ does not warrant the accuracy and reliability of the translations (including but not limited to local regulations, clinical guidelines, terminology, drug names and drug dosages), and is not responsible for any error and/or omissions arising from translation and adaptation or otherwise.

Open access This is an open access article distributed in accordance with the Creative Commons Attribution Non Commercial (CC BY-NC 4.0) license, which permits others to distribute, remix, adapt, build upon this work non-commercially, and license their derivative works on different terms, provided the original work is properly cited, appropriate credit is given, any changes made indicated, and the use is non-commercial. See: http://creativecommons.org/licenses/by-nc/4.0/.

ORCID iDs

Yamato Tsuboi http://orcid.org/0000-0001-5250-8277

Rei Ono http://orcid.org/0000-0002-0176-2870

\section{REFERENCES}

1 Sihawong R, Sitthipornvorakul E, Paksaichol A, et al. Predictors for chronic neck and low back pain in office workers: a 1-year prospective cohort study. J Occup Health 2016;58:16-24.

2 Campos-Fumero A, Delclos GL, Douphrate DI, et al. Low back pain among office workers in three Spanish-speaking countries: findings from the CUPID study. Inj Prev 2017;23:158-64.

3 Vos T, Flaxman AD, Naghavi M, et al. Years lived with disability (YLDs) for 1160 sequelae of 289 diseases and injuries 1990-2010: a systematic analysis for the global burden of disease study 2010 . Lancet 2012;380:2163-96.

4 Nagata T, Mori K, Ohtani M, et al. Total health-related costs due to absenteeism, Presenteeism, and medical and pharmaceutical expenses in Japanese employers. J Occup Environ Med 2018;60:e273-80.

5 Itoh H, Kitamura F, Yokoyama K. Estimates of annual medical costs of work-related low back pain in Japan. Ind Health 2013;51:524-9.

6 Healy GN, Eakin EG, Lamontagne AD, et al. Reducing sitting time in office workers: short-term efficacy of a multicomponent intervention. Prev Med 2013;57:43-8.

7 Clemes SA, O'Connell SE, Edwardson CL. Office workers' objectively measured sedentary behavior and physical activity during and outside working hours. J Occup Environ Med 2014;56:298-303.

8 Nachemson AL. Disc pressure measurements. Spine 1981;6:93-7. 
9 Beach TAC, Parkinson RJ, Stothart JP, et al. Effects of prolonged sitting on the passive flexion stiffness of the in vivo lumbar spine. Spine J 2005;5:145-54.

10 Tissot F, Messing K, Stock S. Studying the relationship between low back pain and working postures among those who stand and those who sit most of the working day. Ergonomics 2009;52:1402-18.

11 Curran M, O'Sullivan L, O'Sullivan $\mathrm{P}$, et al. Does using a chair Backrest or reducing seated hip flexion influence trunk muscle activity and discomfort? A systematic review. Hum Factors 2015;57:1115-48.

12 O'Sullivan K, O'Keeffe M, O'Sullivan L, et al. The effect of dynamic sitting on the prevention and management of low back pain and low back discomfort: a systematic review. Ergonomics 2012;55:898-908.

13 Ognibene GT, Torres W, von Eyben R, et al. Impact of a Sit-Stand workstation on chronic low back pain: results of a randomized trial. $J$ Occup Environ Med 2016;58:287-93.

14 Qaseem A, Wilt TJ, McLean RM, et al. Noninvasive treatments for acute, subacute, and chronic low back pain: a clinical practice guideline from the American College of physicians. Ann Intern Med 2017;166:514-30.

15 Airaksinen O, Brox Jl, Cedraschi C, et al. Chapter 4. European guidelines for the management of chronic nonspecific low back pain. Eur Spine J 2006;15:s192-300.

16 Shariat A, Cleland JA, Danaee M, et al. Effects of stretching exercise training and ergonomic modifications on musculoskeletal discomforts of office workers: a randomized controlled trial. Braz $J$ Phys Ther 2018;22:144-53.

17 Sheahan PJ, Diesbourg TL, Fischer SL. The effect of rest break schedule on acute low back pain development in pain and non-pain developers during seated work. Appl Ergon 2016;53 Pt A:64-70.

18 Hemming K, Taljaard M, McKenzie JE, et al. Reporting of stepped wedge cluster randomised trials: extension of the CONSORT 2010 statement with explanation and elaboration. BMJ 2018;363:k1614.

19 Brown CA, Lilford RJ. The stepped wedge trial design: a systematic review. BMC Med Res Methodol 2006;6:54.

20 Woertman W, de Hoop E, Moerbeek M, et al. Stepped wedge designs could reduce the required sample size in cluster randomized trials. J Clin Epidemiol 2013;66:752-8.

21 Kawashima M, Sano K, Takechi S, et al. Impact of lifestyle intervention on dry eye disease in office workers: a randomized controlled trial. J Occup Health 2018;60:281-8.

22 Hoy D, March L, Brooks P, et al. Measuring the global burden of low back pain. Best Pract Res Clin Rheumatol 2010;24:155-65.

23 Peeler JD, Anderson JE. Reliability limits of the modified Thomas test for assessing rectus femoris muscle flexibility about the knee joint. $J$ Athl Train 2008;43:470-6.

24 Murakami E, Aizawa T, Noguchi K, et al. Diagram specific to sacroiliac joint pain site indicated by one-finger test. $J$ Orthop Sci 2008;13:492-7.

25 Keller S, Bann CM, Dodd SL, et al. Validity of the brief pain inventory for use in documenting the outcomes of patients with noncancer pain. Clin J Pain 2004;20:309-18.
26 Tan G, Jensen MP, Thornby Jl, et al. Validation of the brief pain inventory for chronic nonmalignant pain. J Pain 2004;5:133-7.

27 Uki J, Mendoza T, Cleeland CS, et al. A brief cancer pain assessment tool in Japanese: the utility of the Japanese Brief Pain Inventory-BPI-J. J Pain Symptom Manage 1998;16:364-73.

28 Roland M, Morris R. A study of the natural history of back pain. Part I: development of a reliable and sensitive measure of disability in lowback pain. Spine 1983;8:141-4.

29 Suzukamo Y, Fukuhara S, Kikuchi S, et al. Validation of the Japanese version of the Roland-Morris disability questionnaire. J Orthop Sci 2003;8:543-8.

30 Hill JC, Dunn KM, Lewis M, et al. A primary care back pain screening tool: identifying patient subgroups for initial treatment. Arthritis Rheum 2008;59:632-41.

31 Matsudaira K, Kikuchi N, Kawaguchi M. Development of a Japanese version of the STarT (subgrouping for targeted treatment) back screening tool: translation and linguistic validation. J Musculoskelet Pain Res 2013;5:11-19.

32 Lerner D, Amick BC, Rogers WH, et al. The work limitations questionnaire. Med Care 2001;39:72-85

33 Takegami M, Yamazaki S, Greenhill A, et al. Work performance assessed by a newly developed Japanese version of the work limitation questionnaire in a general Japanese adult population. $J$ Occup Health 2014;56:124-33.

34 Oshima Y, Kawaguchi K, Tanaka S, et al. Classifying household and locomotive activities using a triaxial accelerometer. Gait Posture 2010;31:370-4.

35 Ohkawara K, Oshima Y, Hikihara Y, et al. Real-time estimation of daily physical activity intensity by a triaxial accelerometer and a gravity-removal classification algorithm. Br J Nutr 2011;105:1681-91.

36 Rasmussen CL, Nabe-Nielsen K, Jørgensen MB, et al. The association between occupational standing and sedentary leisure time over consecutive workdays among blue-collar workers in manual jobs. Int Arch Occup Environ Health 2019;92:481-90.

37 Ostelo RWJG, Deyo RA, Stratford P, et al. Interpreting change scores for pain and functional status in low back pain: towards international consensus regarding minimal important change. Spine 2008;33:90-4.

38 Waongenngarm P, Areerak K, Janwantanakul P. The effects of breaks on low back pain, discomfort, and work productivity in office workers: a systematic review of randomized and non-randomized controlled trials. Appl Ergon 2018;68:230-9.

39 Jordan JL, Holden MA, Mason EE, et al. Interventions to improve adherence to exercise for chronic musculoskeletal pain in adults. Cochrane Database Syst Rev 2010:CD005956.

40 Andersen LL. Influence of psychosocial work environment on adherence to workplace exercise. J Occup Environ Med 2011;53:182-4

41 Michishita R, Jiang $\mathrm{Y}$, Ariyoshi D, et al. The practice of active rest by workplace units improves personal relationships, mental health, and physical activity among workers. J Occup Health 2017;59:122-30. 